\title{
Transcriptional and functional consequences of alterations to MEF2C and its topological organization in neuronal models
} \author{
Derek J.C. Tai ${ }^{1-3}$, James F. Gusella ${ }^{1-4,7}$, Sarah Vergult ${ }^{5}$, Michael E. Talkowski ${ }^{1-4,8}$ \\ 1. Center for Genomic Medicine, Massachusetts General Hospital, Boston, MA \\ 2. Program in Medical and Population Genetics, Broad Institute of MIT and Harvard, Cambridge, MA \\ 3. Department of Neurology, Massachusetts General Hospital and Harvard Medical School, Boston, MA \\ 4. Program in Biological and Biomedical Sciences, Harvard Medical School, Boston, MA \\ 5. Center for Medical Genetics, Dept. of Biomolecular Medicine, Ghent University, Ghent, Belgium \\ 6. Division of Genetics and Genomics, Boston Children's Hospital, Boston, MA \\ 7. Department of Genetics, Harvard Medical School, Boston, MA \\ 8. Stanley Center for Psychiatric Research, Broad Institute of MIT and Harvard, Cambridge, MA
}

Kiana Mohajeri ${ }^{1-4^{*}}$, Rachita Yadav ${ }^{1-3^{*}}$, Eva D’haene ${ }^{5}$, Philip M. Boone ${ }^{1-3,6}$, Serkan Erdin ${ }^{1-3}$, Dadi Gao ${ }^{1-3}$, Mariana Moyses-Oliveira ${ }^{1-3}$, Riya Bhavsar ${ }^{1}$, Benjamin Currall ${ }^{1}$, Kathryn O'Keefe ${ }^{1}$, Chelsea Lowther ${ }^{1-3}$, Diane Lucente ${ }^{1}$, Nicholas D. Burt ${ }^{1}$, Monica Salani ${ }^{1-3}$, Matthew Larson ${ }^{1}$, Björn Menten $^{5}$,

"These authors contributed equally to the work

Correspondence: Michael E. Talkowski (mtalkowski@mgh.harvard.edu)

\section{ABSTRACT}

Point mutations and structural variants directly disrupting the coding sequence of $M E F 2 C$ have been associated with a spectrum of neurodevelopmental disorders (NDDs), while recent studies have also implicated altered noncoding regulation of $M E F 2 C$ expression in NDDs. However, the impact of haploinsufficiency of MEF2C on neurodevelopmental pathways and synaptic processes is not well understood, nor are the complex mechanisms that govern regulation of MEF2C. To explore the transcriptional and functional changes associated with coding and noncoding structural variants, we generated an allelic series of 204 isogenic iPSC-derived neuronal cell lines harboring CRISPR-engineered mutations that directly delete predominant isoforms of $M E F 2 C$, as well as deletions to the boundaries of topologically associating domains (TADs) and chromatin loops encompassing MEF2C. We then performed systematic profiling of mutation-specific alterations to transcriptional signatures, regulatory interactions, chromatin contacts, and electrophysiological effects. Our analyses reveal that direct deletion of $M E F 2 C$ causes differential expression of genes enriched for neurodevelopmental and synaptic-associated pathways, accompanied by a significant reduction in synaptic firing and synchrony in neurons. By contrast, we observe robust buffering against MEF2C regulatory disruption upon deletion of a distal $5 q 14.3$ TAD and loop boundary; however, homozygous loss of proximal loop boundary resulted in significant down-regulation of MEF2C expression and significantly reduced electrophysiological activity that was comparable to direct MEF2C disruption. Collectively, our findings demonstrate the functional impact of MEF2C haploinsufficiency in human-derived neural models and highlight the complex interactions of gene regulation and chromatin topology that challenge a priori regulatory predictions of structural variant disruption to three-dimensional genome organization.

\section{INTRODUCTION}

Over the last decade, genetic studies have established of direct gene disruption in NDDs, functional interpretation of haploinsufficiency of MEF2C as a cause of neurodevelopmental noncoding variation remains a considerable challenge ${ }^{23,24}$, though disorders (NDDs) ${ }^{1-12}$. Through microarray analysis, exome and multiple studies have described highly penetrant noncoding genome sequencing, NDDs have been associated with loss-of- mutations across rare NDDs and Mendelian disorders ${ }^{25-27}$. function (LoF) mutations in MEF2C, including protein-truncating One emerging mutational mechanism not captured by exome variants (PTVs), structural variants (SVs) including deletions and genome sequencing is regulatory changes associated and balanced chromosomal abnormalities (BCAs), and broader with three-dimensional (3D) genome organization ${ }^{28-30}$. Early microdeletion of the $5 q 14.3$ locus. Molecular studies of MEF2C glimpses into the intricacies of this architecture demonstrated function have further demonstrated sensitivity to dosage of this the partitioning of chromatin into topologically associating synaptic regulator in mouse models of conditionally modulated Mef2c domains (TADs), and the smaller loops within them. It is presently expression in neural tissue, which display fundamentally altered brain understood that TADs demarcate neighborhoods of long-range development and neuronal activity ${ }^{13-17}$. The extensive complementary regulatory interaction, while loops facilitate punctate enhancer data from human patients and mouse models has implicated MEF2C and promoter connections ${ }^{31,32}$. There are now examples in the LoF mutations as a driver of aberrant neurodevelopment with literature of pathogenic consequences of positional effects varied consequences that include developmental delay, intellectual through the disruption of TADs and loops ${ }^{31,33-35}$, as well as disability, autism spectrum disorder, hypotonia, and epilepsy ${ }^{3,4,10,18-22}$. studies that have demonstrated an uncoupling of topological To date, the mechanisms associated with haploinsufficiency of rewiring and gene expression ${ }^{29,32,36,37}$. These studies collectively $M E F 2 C$ that contribute to these phenotypic presentations have underscore the complexity of long-range regulatory mechanisms not been explored in human neuronal models, which can provide and the significant challenges associated with prediction of insights into signatures of MEF2C-specific changes as well as functional consequences associated with alterations to TAD evidence of transcriptional or functional convergence across NDDs. boundaries and 3D regulatory organization. These studies suggest that the contributions of functional elements are likely In contrast to the abundant molecular studies to be context-specific and require functional modeling to dissect February 2022 (version 1.0) 
bioRxiv preprint doi: https://doi.org/10.1101/2022.02.15.480098; this version posted February 15, 2022. The copyright holder for this preprint (which was not certified by peer review) is the author/funder, who has granted bioRxiv a license to display the preprint in perpetuity. It is made available under aCC-BY 4.0 International license.

bioR $x$ iv Preprint

these diverse regulatory mechanisms of individual loci.

We previously demonstrated through whole-genome sequencing of NDD cases that chromosome $5 q 14.3$ harbored an unusual and genome-wide significant excess of noncoding BCA breakpoints that did not directly disrupt MEF2C but that all occurred within the TAD boundaries encompassing MEF2 $C^{38}$. This distribution of breakpoints in proximity to MEF2C was further supported by microdeletions in NDD cases reported in DECIPHER that apparently did not directly alter the gene locus (at the available resolution of chromosomal microarray) ${ }^{39}$. In considering the landscape of de novo SVs across the $5 q 14.3$ locus in NDD cases, the unifying thread appears to be recurrent distal boundary disruption. Taken together, these data suggest that both direct disruption of MEF2C and alterations to its 3D regulatory architecture may result in comparable molecular mechanisms in NDD cases. Motivated by these findings, we have performed a systematic molecular dissection of the $5 q 14.3$ locus to quantify the transcriptomic and electrophysiological effects of MEF2C LoF in human neural derivatives. Through the generation of an allelic series of CRISPR-engineered human induced pluripotent stem cell (hiPSC)-derived neural stem cells (NSCs) and glutamatergic neurons (iNs), we interrogated the impact of enhancer, TAD boundary, and loop boundary deletion on local genome organization, local expression effects on $M E F 2 C$, and global transcriptional signatures. Our analyses reveal that direct MEF2C alteration results in both transcriptional and functional changes to the synapse. Moreover, we find that disruption of the distal boundary of the MEF2C-containing loop is insufficient to produce indirect $M E F 2 C$ haploinsufficiency, whereas disruption of the proximal boundary of the same $3 D$ structure results in haploinsufficiency of $M E F 2 C$ that is comparable to direct gene disruption. Overall, these data suggest that the effects of direct and indirect MEF2C disruption contribute to cell type-specific alterations on neuronal functions that converge on synaptic deficits in neurodevelopment.

\section{RESULTS}

Haploinsufficiency of MEF2C is associated with altered expression of highly constrained genes in NSCs and synaptic genes in iNs

There is strong evidence for association between microdeletions and LoF point mutations that disrupt MEF2C and a spectrum of NDDs. We therefore first sought to determine the transcriptional changes in early neuronal development stem cells and fully differentiated neurons caused by direct disruption of MEF2C. We generated targeted heterozygous (DEL het) and homozygous (DEL hom) deletions of MEF2C in hiPSCs using dual-guide CRISPR/Cas9 genome editing. Following single-cell isolation and screening, we retained both edited clones and, as matched controls, clones that were exposed to all experimental conditions but were not edited. Six replicates per genotype then underwent differentiation to NSCs and iNs for transcriptional profiling using RNAseq (Figure 1A). MEF2C expression effects were confirmed to be commensurate with zygosity via protein expression analyses (SupplementaryMethods Section 1;SupplementaryFigure1-4).

We performed differential expression analysis using DESeq2 and SVAseq, to account for unknown sources of variation in expression data. In this analysis, differentially expressed genes (DEGs) were selected at Benjamini-Hochberg corrected p-values $(F D R)<0.1$. In NSCs, we observed a strong zygosity-dependent transcriptional response to MEF2C disruption with 371 and 2,196 DEGs in DEL het and DEL hom, respectively (Figure 1B). By contrast, with iNs we observed 742 DEGs following DEL het that were notable for a predominance of upregulated genes and 292 DEGs in DEL hom cells. Nonetheless, we found highly significant overlap and directionally concordant DEGs between DEL het and DEL hom genotypes in both NSCs $(p-$ value $=1.31 \mathrm{e}-39)$ and iNs $(p-$ value $=3.69 \mathrm{e}-36)($ Figure 1C) .

Gene-set enrichment analysis highlighted differential molecular consequences to MEF2C deletion in NSCs and iNs. Haploinsufficiency of MEF2C in NSCs led to dysregulation of genes involved in developmental processes including developmental pattern specification, organ morphogenesis, neurogenesis, and neuron differentiation (Figure 1D). Additionally, DEGs resulting from homozygous loss of MEF2C in NSCs were enriched for LoF constrained genes and gene-sets associated with NDDs from exome sequencing ${ }^{22}$. These LoF and NDD-associated genes are heavily weighted toward genes that display high levels of expression during early neurodevelopment and experience strong negative selection against gene disruptive mutations (Figure 1D). In contrast to NSCs, DEGs identified in iNs were significantly enriched for DEGs observed in forebrain excitatory neurons of an Mef2c knockout mouse model published by Harrington et $\mathrm{al}^{40}$ (Figure 1D). Additionally, DEGs observed in DEL het iNs were enriched for functional terms such as neurogenesis and neuronal differentiation. Homozygous loss of MEF2C in iNs also yielded DEGs that were enriched for synaptic genes (Figure 1D). When comparing these results to data from the BrainSpan project ${ }^{41}$, which identified neural activitydefining gene co-expression modules using 146 samples from 21 fetal to infant developing brains, iN DEGs were enriched for BrainSpan modules associated with synaptic transmission, synaptic maturation, and genes defined as MEF2C binding targets $^{41}$ (modules M13 and M17; Figure 1D). These findings replicated using co-expression modules from a more recent and larger study of 1,230 samples from 48 brains in psychENCODE (Li et al ${ }^{42}$. We observed the module from Li. et al. (ME37), which includes MEF2C, was enriched for DEGs from DEL het iNs (Figure 1D). This module from the psychENCODE study demonstrated expression patterns associated with neuron development and was enriched for genes that converged on associations with neurodevelopmental and neuropsychiatric disorders

Overall, the biological pathways and processes shared across DEGs from NSCs and iNs were strongly enriched for neuronal terms including neurogenesis, neuron differentiation, and regulation of cell differentiation and specifically neuron differentiation (FDR < 0.1). Intriguingly, we also observed that DEGs identified in both NSCs and iNs were enriched for AACTTT and FOXJ2 binding motifs (FDR $<0.1$ ). The AACTTT binding motif has previously been associated with the promoter of MEF2C and enriched at the promoters of genes involved in neurodevelopment and muscle development ${ }^{43,44}$. The FOXJ2 binding motif has similarly been shown to recruit transcriptional activators that function in early developmental stages ${ }^{45}$. Taken together, these data suggest that the expression signatures associated with LoF mutations of $M E F 2 C$ are consistent with perturbations to highly constrained genes broadly involved in transcriptional regulation during early neural development, as well as genes that display distinct expression patterns in later developmental time points and impact neuronal communication and synaptic functions. 
bioRxiv preprint doi: https://doi.org/10.1101/2022.02.15.480098; this version posted February 15, 2022. The copyright holder for this preprint (which was not certified by peer review) is the author/funder, who has granted bioRxiv a license to display the preprint in perpetuity. It is made available under aCC-BY 4.0 International license.

bioR ${ }^{\text {iv }}$ Preprint
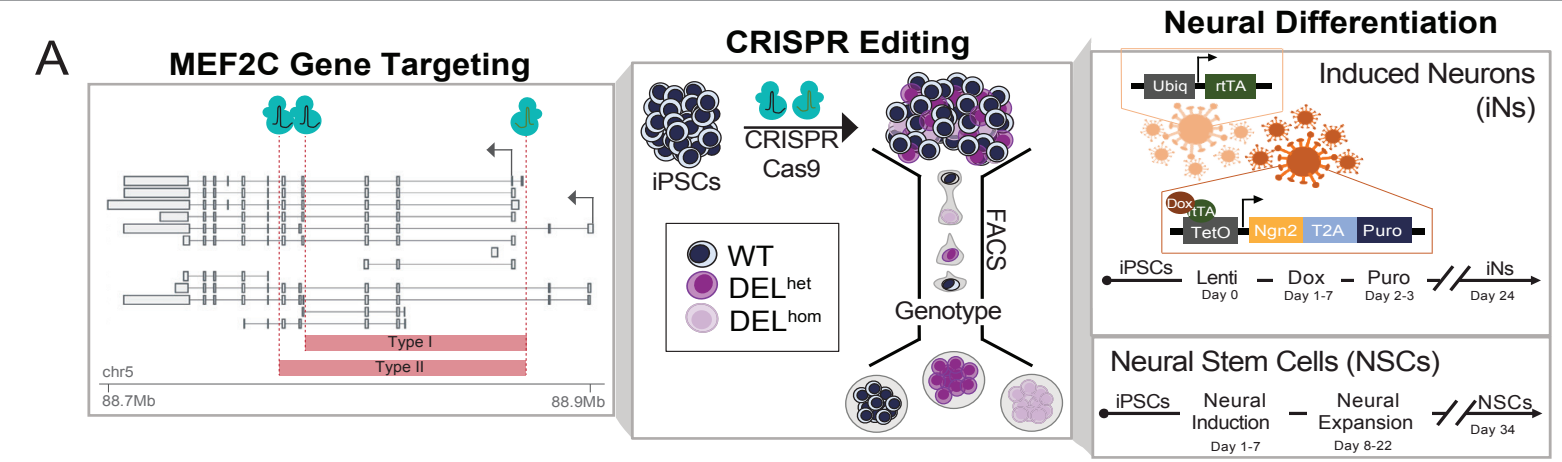

B
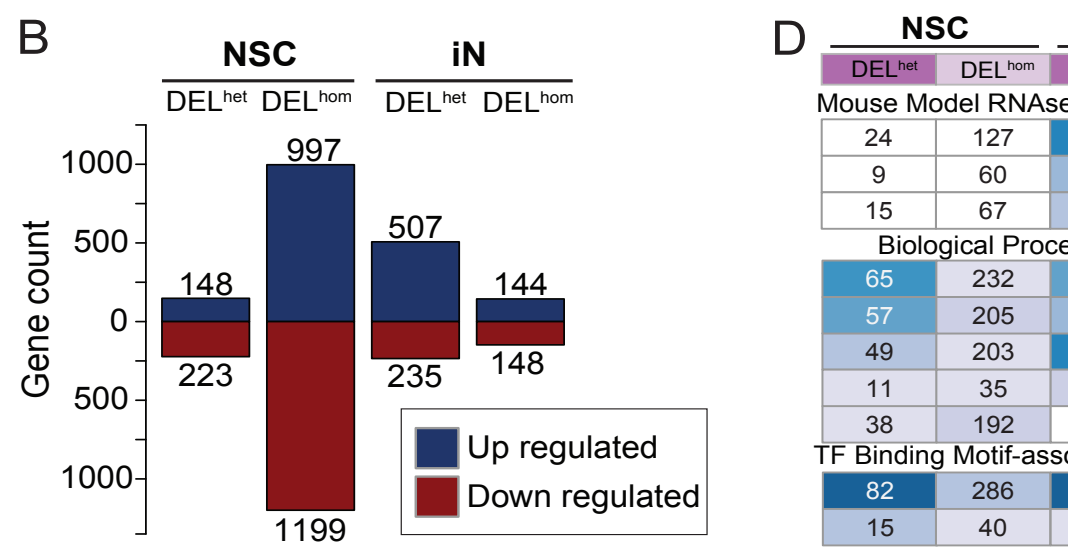

iN Mouse Model RNAseq DEG Validation \begin{tabular}{|c|c|c|c|c|}
\hline 24 & 127 & 93 & 45 & DEGs (All)
\end{tabular} \begin{tabular}{|c|c|c|c|l|}
\hline 24 & 127 & 93 & 45 & DEGs (All) \\
\hline 9 & 60 & 44 & 19 & DEGs (Up reg.) \\
\hline 15 & 67 & 49 & 26 & DEGs (Down reg.) \\
\cline { 1 - 2 } & \multicolumn{3}{c}{ Biological Process Gene Sets } &
\end{tabular}

\begin{tabular}{|c|c|c|c|l|}
\hline 65 & 232 & 114 & 43 & Neurogenesis \\
\hline 57 & 205 & 94 & 35 & Neuron differentiation \\
\hline 49 & 203 & 108 & 34 & Regulation of cell differentiation \\
\hline 11 & 35 & 17 & 9 & Regulation of neuron differentiation \\
\hline 38 & 192 & 68 & 44 & Synaptic genes \\
\hline
\end{tabular}

TF Binding Motif-associated Gene Sets

\begin{tabular}{|c|c|c|c|l|}
\hline 82 & 286 & 163 & 51 & AACTTT motif \\
\hline 15 & 40 & 17 & 11 & FOXJ2 motif \\
\hline
\end{tabular}

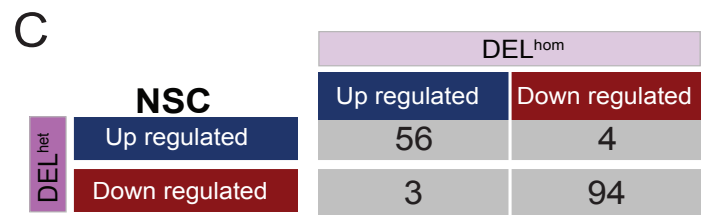

p-value: 1.31e-39

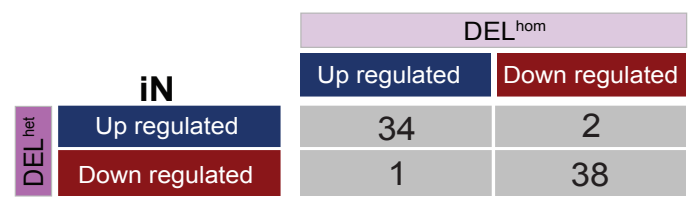

p-value: $3.69 \mathrm{e}-36$

\begin{tabular}{|c|c|c|c|c|}
\hline \multicolumn{5}{|c|}{ NDD-associated and } \\
\hline 64 & 486 & 157 & 53 & LoF \\
\hline 5 & 67 & 17 & 6 & NDD \\
\hline \multicolumn{5}{|c|}{ DNA Binding and Transcription Modules } \\
\hline 11 & 143 & 20 & 7 & $\mathrm{M}^{\ddagger}$ \\
\hline \multicolumn{5}{|c|}{ Synaptic Activity Modules } \\
\hline 15 & 46 & 41 & 18 & M1 \\
\hline 6 & 69 & 8 & 6 & M16 \\
\hline 13 & 57 & 58 & 34 & M17 \\
\hline
\end{tabular}

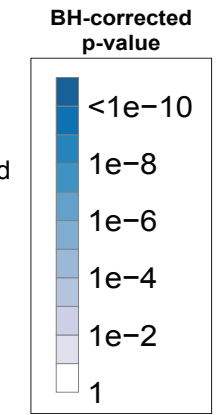

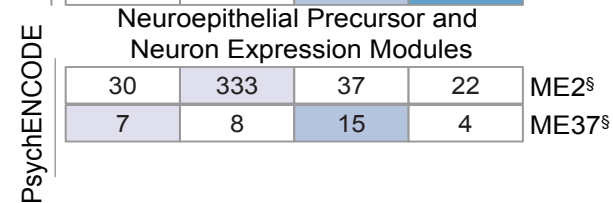

Figure. 1 | MEF2C deletion yields differential expression of genes enriched for neurodevelopmental and synaptic processes.

A. Schematic representation of MEF2C transcripts and location of CRISPR guide RNAs that introduced two independent deletions (Type I and Type II) into hiPSCs. Fluorescence-activated cell sorting (FACS) was then performed, followed by screening of individual clones that identified both heterozygous (DEL het) and homozygous (DEL hom) deletions of MEF2C, along with unedited wild-type (WT) clones that were exposed to identical CRISPR targeting but remained unedited. Following hiPSC growth, neural differentiation was conducted followed by RNAseq. MEF2C CRISPR deletion breakpoints are shown relative to transcripts with $>1$ TPM expression in $\geq 1 \mathrm{GTEx}$ tissue. Arrows represent positions of canonical transcription start sites of MEF2C. B. Differentially expressed genes (DEGs) per cell type and genotype. C. DEG concordance analysis by cell type and genotype group with statistical significance of shared directionality calculated using Fisher's exact test. D. DEG enrichment for gene sets and modules previously published with functional associations with neurological phenotype, synaptic activity, and MEF2C function. Mouse Model RNAseq DEG Validation: Harrington et al, eLife, 2016"0; ; Biological Processes Gene Sets: GSEA msigDB ${ }^{46,47}$, Synaptic genes: Syngo v1.1; MEF2C targets: ENCODE LCL ChIPseq ${ }^{48}$; TF Binding Motif-associated Gene Sets: GSEA msigDB ${ }^{46,47}$; LoF constrained: gnomAD ${ }^{49}$; NDD: Neurodevelopmental Disorder-associated genes, Fu, et al medRxiv"22; DNA Binding and Transcription Modules ( $¥$ ): Parikshak et al, Cell, 2013 ${ }^{41}$; Synaptic Activity Modules ( $)$ ): Parikshak et al, Cell, 201341; Neuroepithelial Precursor and Neuron Expression Modules (§): Li et al, Science, $2018^{42}$.

Altered co-expression of genes in neurodevelopmental and notably enriched for processes of heart morphogenesis ${ }^{13,50-52}$ synaptic pathways associated with deletion of MEF2C.

We next established modules of co-expressed genes in NSCs and RHO GTPase activation ${ }^{53}$ (FDR <0.05), both of which have been described previously in relation to MEF2C function. and iNs using weighted gene co-expression network analysis (WGCNA; Figure 2). In NSCs, four co-expression modules had an eigengene that correlated significantly with MEF2C dosage: significantly up-regulated only in DEL het NSCs and was enriched with an eicon violet $(P=1 e-5)$, bisque4 $(P=5.6 e-5)$, yellow4 $(P=8.8 e-3)$, and for terms related to synapse assembly and organization. darkslateblue $(P=1.2 \mathrm{e}-3)$ (Figure 2A, C, E). Yellow4 genes, which showed increased expression with MEF2C loss, were Co-expression analysis in iNs isolated two modules whose 
bioRxiv preprint doi: https://doi.org/10.1101/2022.02.15.480098; this version posted February 15, 2022. The copyright holder for this preprint (which was not certified by peer review) is the author/funder, who has granted bioRxiv a license to display the preprint in perpetuity. It is made available under aCC-BY 4.0 International license.

bioR $x^{i v}$ Preprint
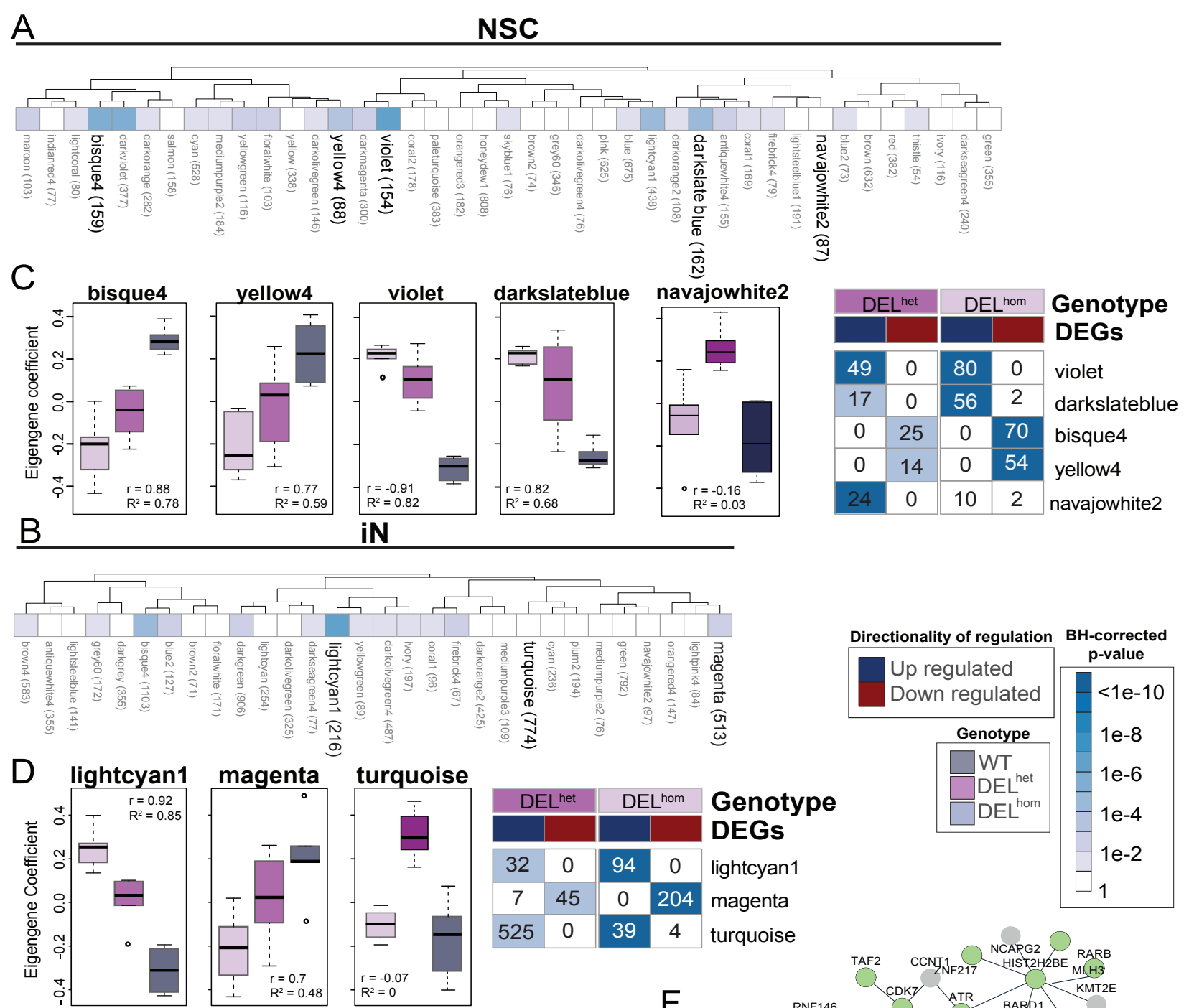

E
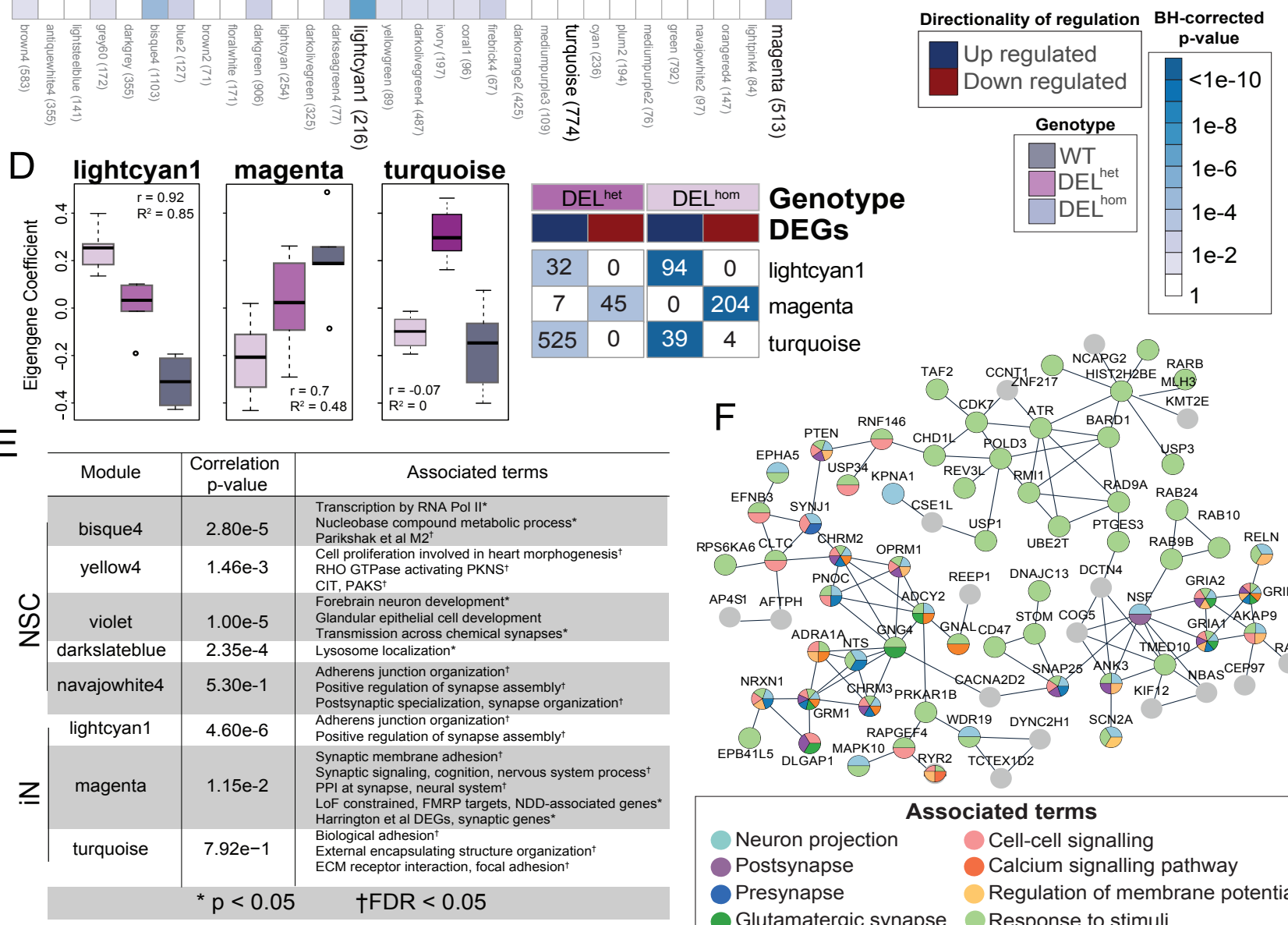
bioRxiv preprint doi: https://doi.org/10.1101/2022.02.15.480098; this version posted February 15,2022 . The copyright holder for this preprint (which was not certified by peer review) is the author/funder, who has granted bioRxiv a license to display the preprint in perpetuity. It is made available under aCC-BY 4.0 International license.

bioRxiv Preprint

were enriched for DNA damage repair-associated nucleotide patch replacement (FDR <0.01). In addition to having an essential role in cardiac and neurodevelopment, previous reports have also described MEF2C as serving lineage specific roles in regulation of DNA damage repair ${ }^{54,55}$. The genes co-expressed in the magenta module revealed a far greater emphasis on alterations to synaptic functions, being enriched for synaptic membrane adhesion, synaptic signaling, and protein-protein interactions at the synapse (FDR $<0.01)$. The encoded proteins $(n=513)$ were mapped to a PPI network using StringDB ${ }^{56,57}$ in Cytoscape ${ }^{58-60}$, resulting in a network with 150 members (confidence score $>0.7$, evidence=experimental/database, p-value < 1e-16). The proteins from 76 genes contained within module magenta formed a network associated with neuronal and synaptic terms (Figure 2F). Module magenta also included genes associated with monogenic forms of epilepsy and other NDDs, such as GRIN2A, SCN2A, and GRIA2, providing evidence of molecular convergence for these genotypically distinct but strongly synaptic activity-associated disease genes ${ }^{61-63}$. MEF2C direct disruption yields changes to synaptic firing and synchrony in human neural models as measured by multi-electrode array (MEA).

Given the strong transcriptional changes associated with deletion of MEF2C in hiPSC-derived neurons that converged on synaptic activity, we sought to functionally validate this association by defining electrophysiological changes in neurons using multi-electrode array (MEA; Figure 3, Supplementary Section 2.1, Supplementary Figures 5-7). We differentiated heterozygous and homozygous MEF2C lines and matched controls to iNs and observed a statistically significant (based on t-test) down-regulation of synaptic activity in iNs. We observed statistically significant reductions in firing rate relative to wildtype for both $\mathrm{DEL}^{\text {het }}(26 \%, \mathrm{P}=1.4 \mathrm{e}-5)$ and $\operatorname{DEL}^{\text {hom }}(31 \%, P=1.3 e-2)$ (Figure $3 A$ ). We also observed statistically significant reductions to spike count relative to wild-type for both DEL het $(17 \%, P=6.7 e-3)$ and $D^{2} L^{\text {hom }}(48 \%, P=5.1 e-5)$ (Figure 3B). Additionally, we observed statistically significant reductions to synchrony, a measure of uniformity of neuronal firing bursts, relative to wildtype for both $D E L^{\text {het }}(32 \%, P=7.1 \mathrm{e}-3)$ and $D E L^{\text {hom }}$ (73\%, $P=9.6 e-9)$ (Figure $3 \mathrm{C}$ ). While we observed clear changes in firing rate, neither $D E L^{\text {het }}(P=0.68)$ nor $D E L^{\text {hom }}(P=0.30)$ loss of MEF2C resulted in significant changes to network burst oscillation (Figure 3D). These data provided a complementary measure of MEF2C direct disruption resulting in altered synaptic activity.

Dissecting the three-dimensional chromatin topology and regulatory interactions within the $5 q 14.3$ locus

Our prior analyses from whole genome sequencing of individuals with NDDs harboring $B C A s^{38}$, and other recent studies ${ }^{1,64-69}$, have suggested that cis-regulatory disruption by noncoding SVs may underlie NDD phenotype association within the $5 q 14.3$ locus beyond direct LoF MEF2C mutation. Collectively, these seven studies have reported CNV and BCA breakpoints 200-500kb distal to $M E F 2 C$ in 13 distinct cases presenting with phenotypes consistent with MEF2C haploinsufficiency such as NDD, epilepsy, and hypotonia. As a result, enhancer-promoter decoupling by disruption to $3 \mathrm{D}$ chromatin organization has emerged as a mechanistic hypothesis for indirect MEF2C disruption. We therefore performed a comprehensive and systematic dissection of the TAD and loop organization of the $5 q 14.3$ region in human neural models. From analyses of existing 2D elements enhancers as well as 3D element like boundaries and structural protein ChIP annotations (CTCF and SMC3) from published datasets ${ }^{31,70}$, we defined both 2D and 3D elements with evidence for a role in MEF2C regulation (Supplementary Section 3.1, Supplementary
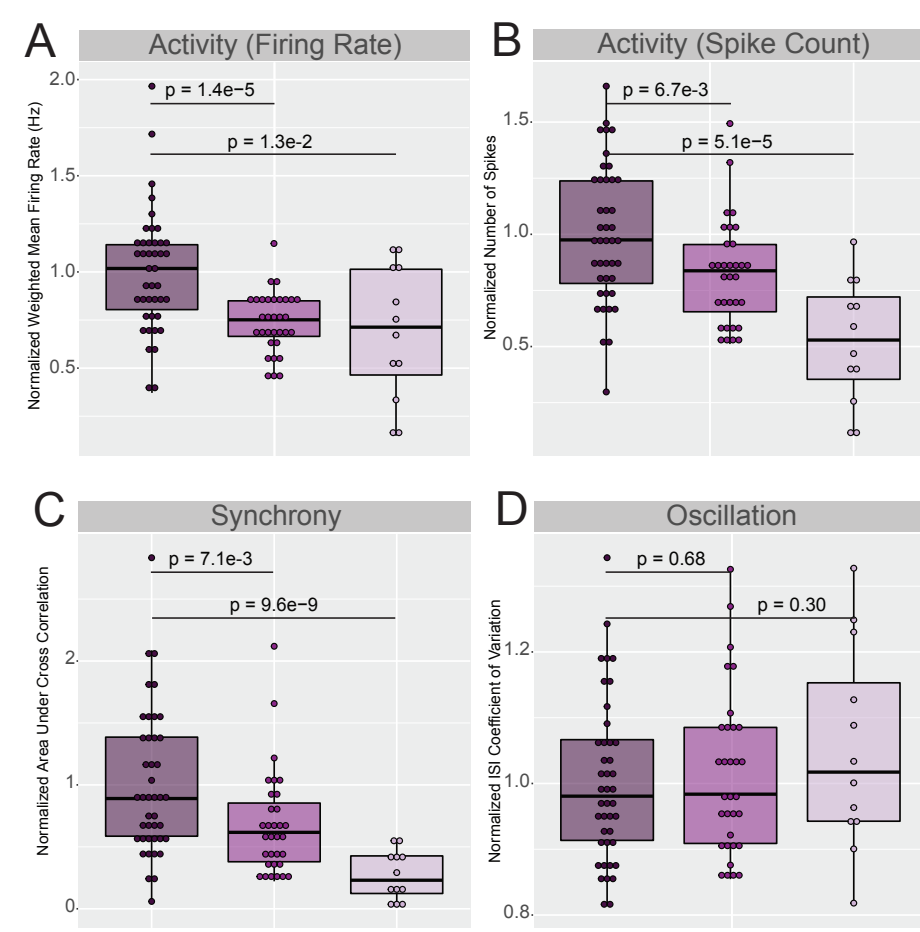

\section{WT}

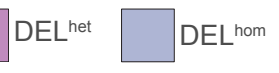

Figure 3 | Deletion of MEF2C is associated with reduced synaptic activity and synchrony in neurons.

Each datapoint represents a single replicate well. Each resultant measurement was normalized per well against the wild-type mean per plate and normalized data points from two replicate plates plotted. A. Normalized weighted mean firing rate $(\mathrm{Hz})$ (Firing Rate). B. Normalized number of spikes (Spike Count). C. Normalized area under cross correlation (Synchrony). D. Normalized ISI (interspike interval) coefficient of variation (Oscillation). P-values calculated using t-test against normalized wells per genotype.

Figures 8-9). We then sought to determine the overarching 3D functional architecture responsible for orchestrating gene-enhancer interactions by generating an allelic series of deletions targeting 2D and 3D functional elements within the 5q14.3 locus (Figure 4).

We annotated topological structures from LCLs using $\mathrm{Hi}-\mathrm{C}$ data and integrated CTCF and SMC3 binding sites indicated by LCL ChIP-seq ${ }^{31,70}$. Dual guide CRISPR/Cas9-based genome editing guides were then designed to engineer a series of deletions of the candidate 3D elements as outlined in Figure 4. We targeted deletion of four genomic sites, including the proximal and distal boundaries of the MEF2C-containing loop as the key experimental edits (referred to as "Proximal Boundary" and "Distal Boundary", respectively), and two 'negative control' edits, namely the boundary of the TAD adjacent to MEF2C ("Adjacent TAD"), and the genomic sequence spanning the MEF2C-containing and adjacent TAD with no occupied CTCF binding sites in 133 cell/tissue samples from ENCODE ("Separator"). We focused on this MEF2C loop structure as opposed to the larger TAD given that it was largely cell type invariant in both the above resources and additional cell types, as well as the strength of contact with MEF $2 \mathrm{C}$ and its higher resolution map of the 3D organization encompassing the MEF2C-relevant enhancers (Supplementary Section 3.1, Supplementary Figures 8-9). Differentiated hiPSC-derived iN and NSC CRISPR 
bioRxiv preprint doi: https://doi.org/10.1101/2022.02.15.480098; this version posted February 15,2022 . The copyright holder for this preprint (which was not certified by peer review) is the author/funder, who has granted bioRxiv a license to display the preprint in perpetuity. It is made available under aCC-BY 4.0 International license.

bioR $x^{i v}$ Preprint

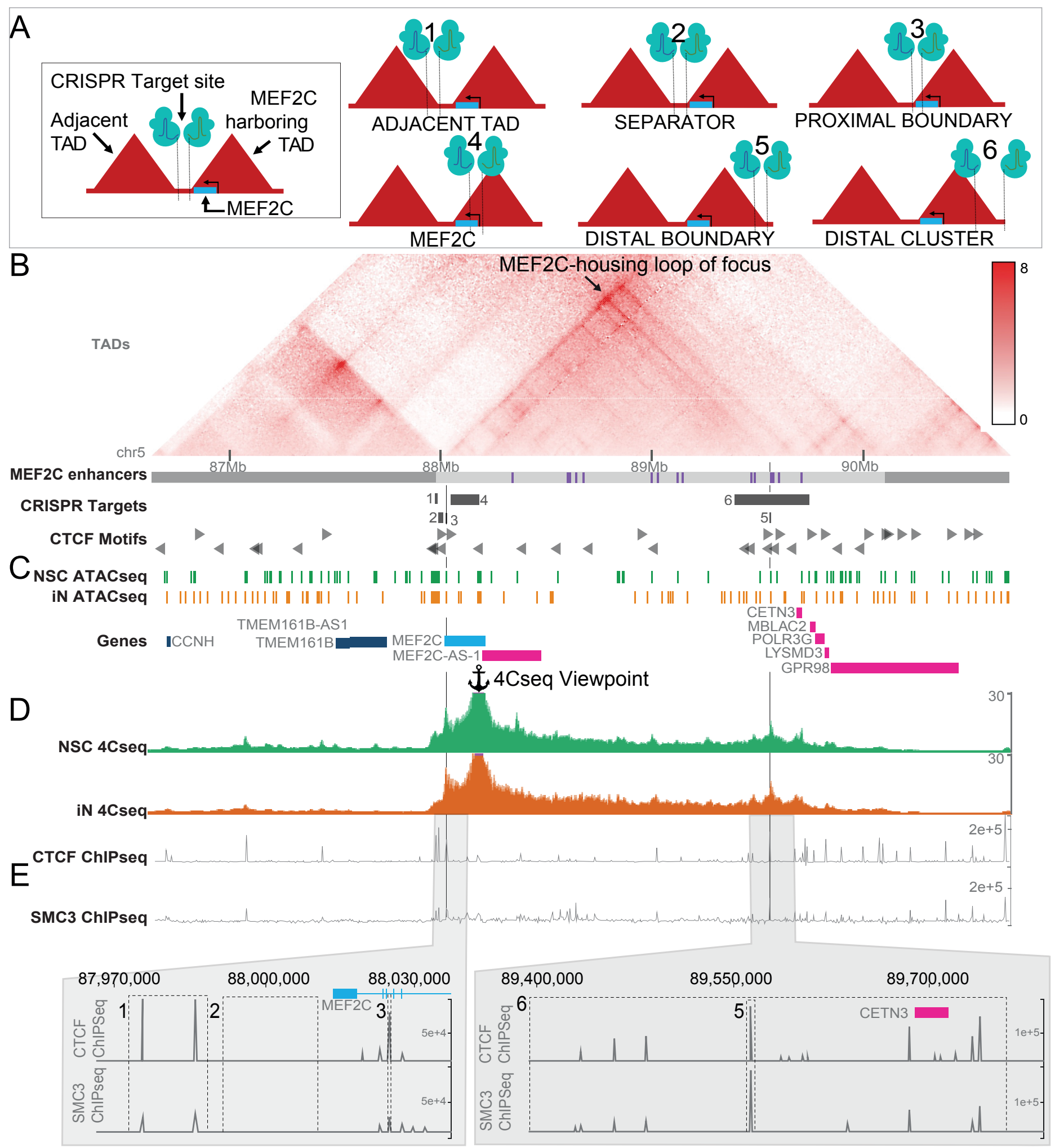

Figure 4 | Design of iPS CRISPR deletion models.

A) Depiction of CRISPR targets relative to MEF2C and local 3D structures. B) Knight-Ruiz normalized Hi-C map from GM12878 wildtype LCLs shown $^{31}$. Local TAD annotations ${ }^{70}$ and putative MEF2C enhancers ${ }^{71}$ denoted as grey and purple bars respectively. Black arrow within the Hi-C map and vertical grey lines highlight the MEF2C-housing loop of focus targeted in this study. C) Open chromatin regions identified by ATACseq in wildtype NSCs and iNs. D) Aggregate contacts from 4Cseq in wildtype NSCs and iNs shown relative to the viewpoint within the MEF2C promoter. E) Deletion positions of 3D topology boundaries guided by ChIPseq for structural proteins CTCF and SMC3 in GM12878 LCLs.

models were generated for each of these four deletion models using six replicates per DEL het and DEL hom genotype, as well as six control clones that were exposed to the CRISPR conditions but not edited (as described in Materials and Methods). Differentiation of these iPSCs established 204 individual neuronal lines representing systematic disruption to functional elements within the $5 q 14.3$ locus (Supplementary Section 3; Supplementary Figures 14-20). 
bioRxiv preprint doi: https://doi.org/10.1101/2022.02.15.480098; this version posted February 15, 2022. The copyright holder for this preprint (which was not certified by peer review) is the author/funder, who has granted bioRxiv a license to display the preprint in perpetuity. It is made available under aCC-BY 4.0 International license.

bioR $x$ iv Preprint

Distal Boundary deletion does not result in marked change to MEF2C expression.

We observed that DEL het and DEL hom of the Separator and Adjacent TAD boundary deletions resulted in no substantial changes in MEF2C expression or to contacts between the MEF2C promoter and published enhancers of MEF $2 \mathrm{C}^{71}$ based on UMI-4C, as expected (Figure 5, Supplementary Section 3.6, Supplementary Figures 22-23, 26-29).

The Distal Boundary deletion served to directly test the necessity of $3 \mathrm{D}$ chromatin topology for robust expression of $M E F 2 C^{1,38,64-67}$. Surprisingly, deletion of this site resulted in no change in MEF2C protein expression and a slight increase (25\% compared to wildtype) in mRNA expression for DEL het in NSCs (log2FC $=0.33$; FDR $<0.1$ when correcting for multiple testing within the TAD, Figure 5; Supplementary Figure 23). Aside from MEF2C, we also considered expression of genes expressed in NSCs or iNs that were located within either the MEF2C-containing TAD, the TAD directly proximal, or the TAD directly distal, which we refer to herein as "local genes", using TADs as defined by Dixon et $\mathrm{al}^{70}$. 15 genes (not including MEF2C) met this criterion for local gene inclusion in NSCs, while 14 were considered in iNs given that MEF2C-AS1 was not expressed in this cell type. We observed no altered expression of any local genes aside from MEF2C in Distal Boundary deletion NSCs. To further explore this finding, we employed allele-specific UMI-4C anchored at the MEF2C promoter (Figure 5, Supplementary Section 3.6, Supplementary Figures 26). These analyses identified an increase in significant (FDR<0.1) contacts between the MEF2C promoter and two separate $6 \mathrm{~kb}$ windows harboring CTCF binding sites immediately distal to the deletion position, suggesting CTCF motif redundancy may buffer deletions to canonical 3D boundary elements, preventing strong dysregulatory effects. In DEL ${ }^{\text {het }}$ matched iNs with Distal Boundary deletions, no significant expression or contact changes were observed for $M E F 2 C$ or any other local genes.

To further evaluate this largely negative result, we tested the hypothesis of regulatory element redundancy buffering against pronounced expression effects on MEF2C. We did so by deleting what we referred to as the Distal Cluster, a $354 \mathrm{~kb}$ region that includes six annotated enhancers (e11-e16), five directly oriented CTCF binding site motifs, the Distal Boundary, and one local gene, CETN3 (Figure 4). We note that the generation of this large CRISPR deletion was performed directly in NSCs (by comparison to the hiPSC stage prior to differentiation for all other models), and is thus a technically distinct validation experiment rather than directly comparable to the models in the initial hypothesis test. Nonetheless, in these analyses we observed no significant differential expression of MEF2C upon DEL het deletion of the Distal Cluster region when compared to matched wildtypes in either NSCs or NSC-derived iNs (Supplementary Figures 23-24). Considering UMI-4C data from the viewpoint of the MEF2C promoter in these lines, we observed a significant $(F D R<0.1)$ increase in contacts with four sites within $5 q 14.3$ alongside a contiguous increase with the $320 \mathrm{~kb}$ region immediately distal to the deleted region in NSCs. Considering local genes not directly disrupted by the deletion, we observed a cascade of significant (FDR $<0.1$ ) dysregulation of $8 / 15$ genes in $5 q 14.3$ locus in iNs, with no genes significantly dysregulated in matched NSCs. Together, these analyses suggest that deletion of the distal boundary of the MEF2C encompassing loop is insufficient to indirectly dysregulate this NDD gene.

Proximal Boundary deletion yields reduction of MEF2C expression and synaptic activity in iNs.

In contrast to the weak or largely negative results observed for deletion of the Distal Boundary, deletion of the Proximal Boundary, which is located in an intron of $M E F 2 C$, had marked effects on the gene's expression that appeared to be genotype and cell-type dependent. NSCs harboring DEL het or DEL hom of the Proximal Boundary did not display differential expression of $M E F 2 C$, though protein expression was significantly reduced in $\mathrm{DEL}^{\text {hom }}$ (49\% relative to controls; $\mathrm{p}$-value $=5.7 \mathrm{e}$ 4, Supplementary Figure 22-23). These DEL hom NSCs also displayed differential contacts with the MEF2C promoter at three sites, two of which were significantly increased and one significantly decreased (locally corrected FDR<0.1). Moreover, homozygous deletion of the Proximal Boundary in iNs resulted in pronounced down-regulation of $M E F 2 C$, five genes localized homozygous deletion of the Proximal Boundary in iNs resulted in pronounced down-regulation of MEF2C, five genes localized to the MEF2C-containing TAD, and three genes within the TAD proximal to the MEF2C-containing TAD (locally corrected FDR $<0.1$; Figure 5). This consistent down-regulation thus extended up to $3 \mathrm{Mb}$ from the site of the deletion, and was also dosagedependent as DEL hom deletion resulted in significantly reduced expression compared to unedited and DEL het cells (Figure 5). These positional effects were particularly strong for $M E F 2 C$, and DEL hom deletion largely recapitulated the reduction observed with heterozygous direct gene deletion (e.g. $45 \%$ reduction, locally corrected FDR $=1.9 \mathrm{e}-9$, genome-wide FDR $=6.3 \mathrm{e}-8$ ) (Figure 5).

We next explored transcriptional and functional commonalities between direct MEF2C disruption and indirect expression reduction by Proximal Boundary deletion. We observed shared DEGs from direct MEF2C disruption in both NSCs and iNs with Proximal Boundary deletion (Figure 6). We also observed shared functional pathways in iNs related to synaptic activity, neurodevelopment, and neural differentiation between these distinct coding and noncoding functional mutations (Figure 6). The Proximal Boundary DEL hom and direct MEF2C deletion iN DEGs also shared significant enrichment of terms such as axon development alongside sharing an enrichment for genes with promoter containing the sequence motif AACTTT, a binding motif in genes involved in neurodevelopment and muscle development ${ }^{43,44}$. Given our demonstration of shared neuronal pathways between direct MEF2C deletion and Proximal Boundary deletion lines, we next tested whether this noncoding regulatory Proximal Boundary deletion replicated the synaptic deficits observed with MEF2C deletions. From these analyses, we observed a similarly strong reduction in synaptic activity for both DEL het and DELom iNs when compared to matched wildtype clones over the differentiation time course measurements (Figure 6; Day31 - Day43). The pattern and significance detected for spike number and number of bursts over time was also consistent with the MEF2C deletion MEA time course experiment, suggesting a reproducible synaptic phenotype associated with both direct deletion and noncoding regulatory alterations to the MEF2C locus (Supplementary Section 3.6, Supplementary Figures 30-32).

\section{DISCUSSION}

We present an initial survey of the transcriptional and electrophysiological consequences of $M E F 2 C$ gene deletion and indirect regulatory changes introduced by alterations to $5 q 14.3$ TAD and loop chromatin topology in human-derived in vitro neural models. Direct LoF mutations (PTVs, deletions, translocations) within MEF2C have repeatedly been associated 
bioRxiv preprint doi: https://doi.org/10.1101/2022.02.15.480098; this version posted February 15, 2022. The copyright holder for this preprint (which was not certified by peer review) is the author/funder, who has granted bioRxiv a license to display the preprint in perpetuity. It is made available under aCC-BY 4.0 International license.

bioR $x$ iv Preprint

\begin{tabular}{|c|c|c|c|c|c|}
\hline $\begin{array}{l}\text { RASA1 M MEF2C } \\
\text { TMEM161B-AS1 } \\
\text { CCNH I } \\
\text { TMEM161B } \\
\text { MEF2C-AS1 }\end{array}$ & $\begin{array}{l}\text { MBLAC2 } \\
\text { POLR3G } \\
\text { LYSMD3 } \\
\text { CETN3! } \\
\text { GPR98 } \\
\text { ARRDC3! }\end{array}$ & $\begin{array}{l}\text { FAM172A } \\
\text { NR2F1I } \\
\text { KIAA0825 } \\
\text { ANKRD32 }\end{array}$ & $\begin{array}{l}\text { RASA1 M MEF2C } \\
\text { TMEM161B-AS1 } \\
\text { CCNH I } \\
\text { TMEM161B } \\
\text { MEF2C-AS1 }\end{array}$ & $\begin{array}{l}\text { MBLAC2 } \\
\text { POLR3G } \\
\text { LYSMD3" } \\
\text { CETN3! } \\
\text { GPR98 } \\
\text { ARRDC3। }\end{array}$ & $\begin{array}{l}\text { FAM172A } \\
\text { NR2F1I } \\
\text { KIAA0825 } \\
\text { ANKRD32 }\end{array}$ \\
\hline
\end{tabular}

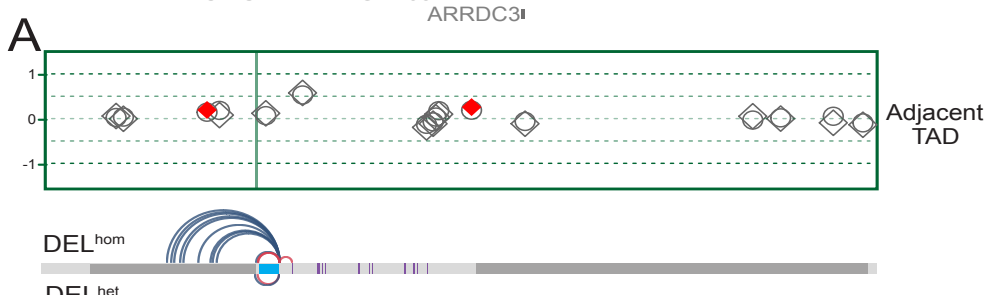

DEL het

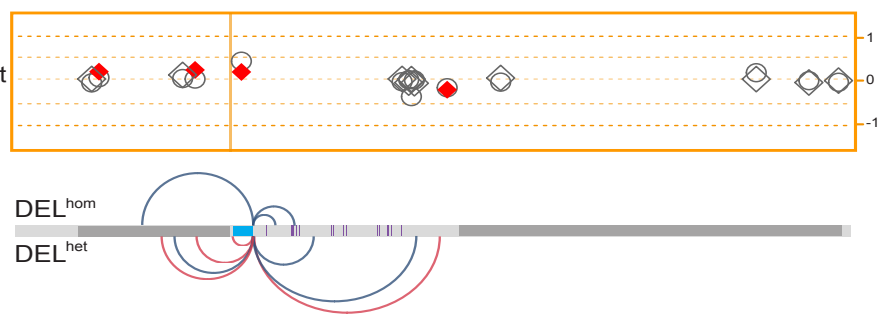

B
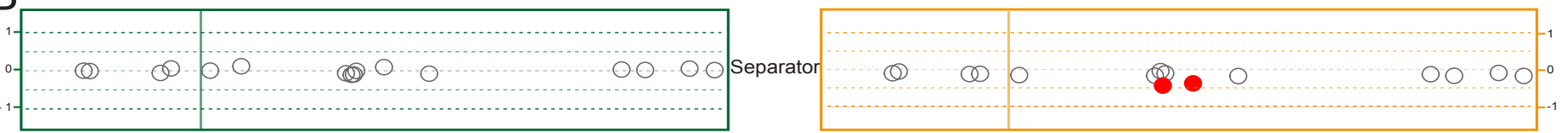

DEL ${ }^{\text {het }}$

DEL $L^{\text {het }}$
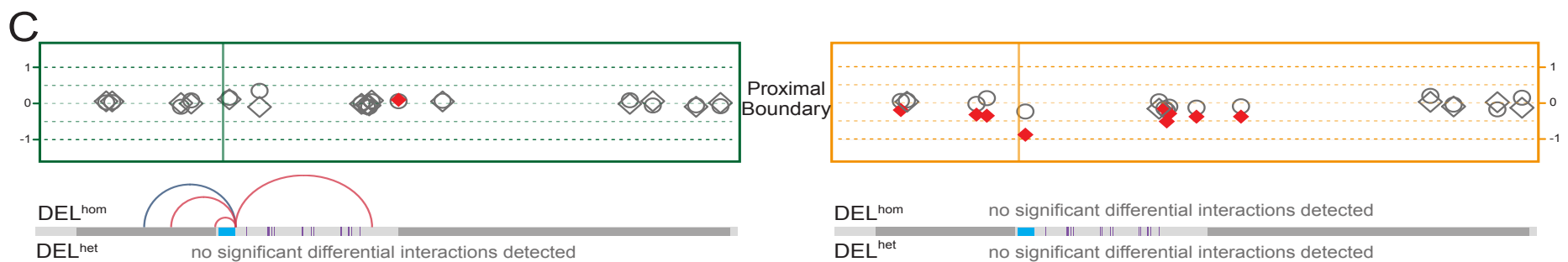

DEL het no significant differential interactions detected

DEL hom no significant differential interactions detected

DEL $^{\text {het }} \quad$ no significant differential interactions detected

$\mathrm{D}$

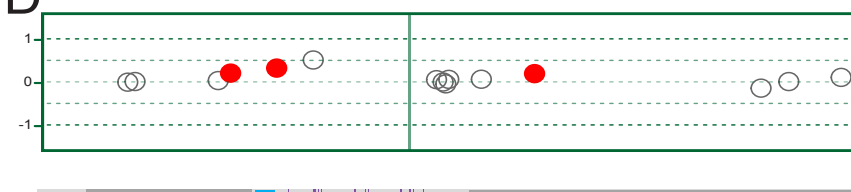

DEL het
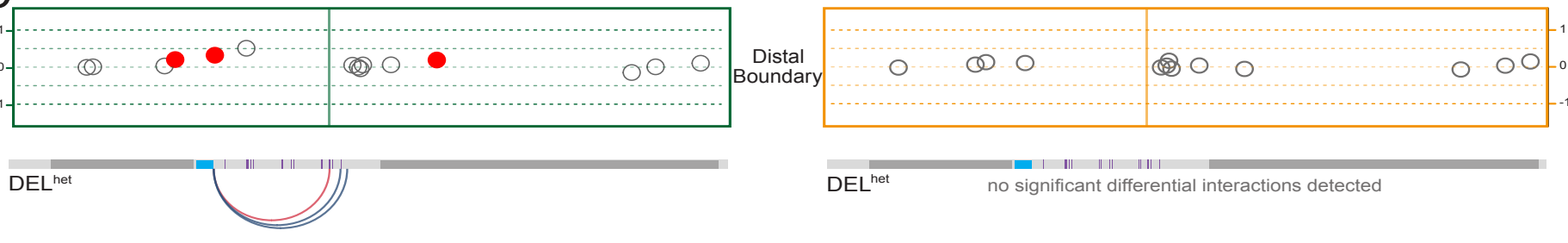

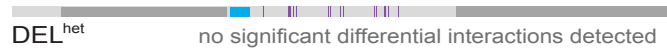

E
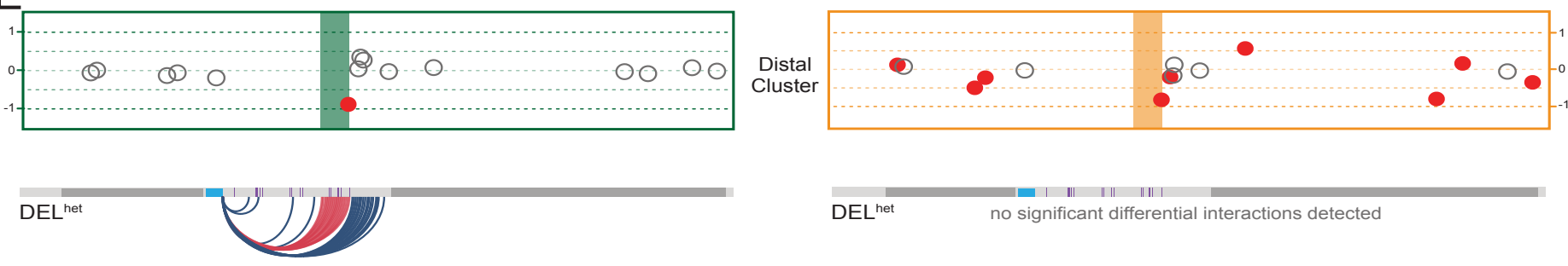

DEL het no significant differential interactions detected

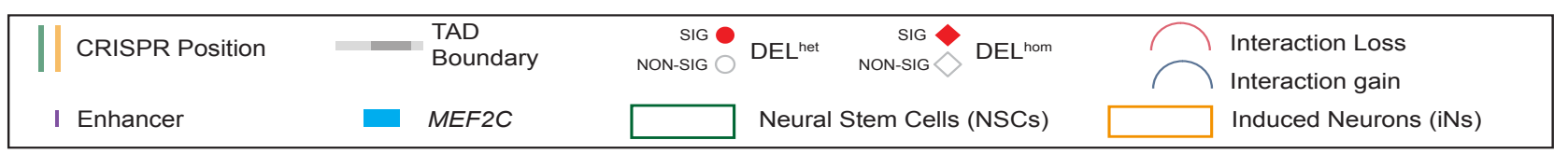

Figure 5 | Resultant MEF2C expression and long-range contact changes from 5q14.3 non-coding element disruption cannot be predicted a priori based on cell type, genotype, or functional element type.

$Y$ axis shows Log2 fold change of differential expression for MEF2C and 15 local genes as defined by their inclusion within the MEF2C-containing TAD, TAD immediately proximal, or distal. The significantly differentially expressed (locally corrected FDR<0.1) genes in NSCs and iNs are shown in red and non-significant genes shown outlined in grey. Differential expression of each gene is annotated by a circle for DEL ${ }^{\text {het }}$ deletions (all CRISPRs) and a diamond for DEL hom deletions (able to be generated in a subset of CRISPRs). Position of CRISPR deletion annotated by vertical line. Differential contacts with the MEF2C promoter in each CRISPR line relative to matched wild-type indicated by curved lines (Supplementary figure 25-29). Lines included for significant contact changes with FDR<0.1 based on a sliding window of $5 \mathrm{~kb}$ with $200 \mathrm{bp}$ step. Note: MEF2C-AS1 is not expressed in iNs. 
with NDD risk. ${ }^{2,3,5,6,10,65,66,72-75}$ A variety of murine knockout models, measured by MEA, suggest shared functional consequences including conditional knockout of MEF2C in varying neural cell from direct and indirect MEF2C disruption. However, the deletion types and developmental time points, have collectively suggested of the Distal Boundary did not recapitulate these results. While a role for $M E F 2 C$ in neuronal function, ${ }^{13,14,16,40}$ but no studies the data are therefore unambiguous of a localized enrichment have evaluated MEF2C LoF mutations in human-derived cell of disease associated noncoding SV breakpoints spanning the lineages. In the study presented here, we engineered an allelic MEF2C-containing 3D organization, the models that displayed series of CRISPR mutations within MEF2C and its putatively the greatest transcriptional and functional consequences were not regulatory $3 \mathrm{D}$ functional elements, and demonstrated that both those that were predicted a priori based on the localization of the direct and indirect alterations to MEF2C expression converge SV breakpoints alone. The noncoding regulatory mechanisms that on themes related to neurodevelopment and synaptic activity.

NDD risk in this region thus remain elusive and appear to Recently, specific studies that have focused on the do not result in the same degree of genome topological rewiring knockout of SMC3 in human cell models ${ }^{29}$, genome as would be predicted from the translocations and inversions rearrangements in drosophila ${ }^{36}$, and TAD disruption at the Shh observed in the BCA cases, and the functional changes observed locus $^{76}$ have demonstrated the uncoupling of regulatory changes are not consistent across cell types. These analyses illustrate associated with gene expression and alterations to long-range the highly complex regulatory architecture of alterations to chromatin contacts. Our data further support those studies, as chromatin topology and emphasize the significant challenges for we observed alterations to $3 \mathrm{D}$ topological organization that computational prediction of the regulatory features of $S V s^{78-80}$. occur without concomitant changes in gene expression. These

demonstrations are in contrast to the hallmark examples of TAD We present the first CRISPR engineered isogenic allelic series disruption underlying dysregulation of a gene associated with a of MEF2C disruption in human-derived in vitro models and human disease phenotype $3^{1,33-35}$. Most of the CRISPR models functional characterization of resultant transcriptional and targeting $2 \mathrm{D}$ and $3 \mathrm{D}$ noncoding element deletions generated electrophysiological effects. These studies also uniquely dissect in this study demonstrated modest or no functional impact on the parallel consequences of direct and indirect alterations to expression of MEF2C and genes encompassed within the MEF2C by cis-regulatory disruption, revealing that some noncoding adjacent TADs. Furthermore, most CRISPR models also did not mutations can recaptiulate synaptic deficits and transcriptional result in major changes to long-range contacts with the MEF2C signatures observed from direct deletion of this gene underlying promoter. We did note some robust changes in contact patterns the well-established $5 q 14.3$ microdeletion syndrome. While direct from UMI-4C conducted in our NSC CRISPR lines, but most deletion of MEF2C revealed functional changes in human neurons observed differential contacts did not engage previously validated that were consistent with previous studies in mouse models ${ }^{14-17,40}$, enhancers of MEF2C. These findings further illustrate the our results following SV alteration to 3D organization of 5q14.3 complexity associated with the dynamic interactions that result underscore the complexity of regulatory interactions at this locus, from SV alterations of 3D topology and regulation of gene function. and more broadly in defining the features associated with the functional impact of noncoding SVs genome-wide. These data Our approach weighted elements contributing to 3D genome clearly demonstrate that alteration to annotated boundaries of 3D organization given the previous reports highlighting TAD disruption regulatory architecture encompassing established human disease as a putative indirect cause of MEF2C haploinsufficiency ${ }^{38,65}$. In genes is insufficient evidence to presume alterations to gene our most recent WGS study of 406 individuals with developmental regulation or phenotypic impact. Future in silico, in vitro, and in disorders that harbored a BCA, as well as 304 BCAs from control vivo studies targeting more loci and classes of SVs with designs individuals, the $5 q 14.3$ locus continues to display a genome-wide that either recapitulate case rearrangements or are agnostic to significant enrichment of noncoding SV breakpoints that localize regulatory element type will be necessary to further expand these distal to MEF2C. The homozygous Proximal Boundary deletion findings into systematic analysis of the features associated with caused a two-fold reduction of MEF2C expression that was 3D genome architecture and noncoding disease association. comparable to haploinsufficiency resulting from heterozygous deletion. The absence of a coding SNP within MEF2C prevented the determination of whether up-regulation of $M E F 2 C$ on the wild-type allele prevented observed differential expression in heterozygous deletion of Proximal Boundary lines. This region has been recently reported as a cis-regulatory site that was demonstrated to reduce MEF2C expression following CRISPRi in $\mathrm{K} 562$ cells $^{77}$. The shared enrichment of gene sets related to neurogenesis and neuronal differentiation between both Proximal Boundary and MEF2C DEGs demonstrates transcriptome dysregulation by both direct and indirect MEF2C disruption that converge upon biological processes of relevance to NDDs. We also observe significant overlap between indirect disruption of MEF2C by Proximal Boundary deletion in NSCs and direct $M E F 2 C$ disruption in iNs, suggesting dysregulatory effects may involve temporal or cell type specific dysregulation. Furthermore, the shared enrichment of genes with AATCCC binding motifs within their promoters, which was previously associated with genes involved in neuronal differentiation, including MEF2C, alongside the comparable reductions in synaptic activity as

\section{METHODS \& SUPPLEMENTARY INFO}

Detailed methods and supplementary information for this manuscript have been provided in a separate document, which will be linked directly from bioRxiv.

\section{ACKNOWLEDGMENTS}

We would like to thank Maris Handley and the staff of the Harvard Stem Cell Institute FACS core at Massachusetts General Hospital for helpful discussion and technical assistance with cell sorting for iPS clone generation. We would also like to thank Lies Vantomme for excellent technical assistance.

This research was supported by grants from the National Institutes of Health: P01GM061354, R01HD096326, R01MH115957, R01MH123155, U01HG011755, R03HD099547, R01NS093200, K08NS117891, T32GM007748, G044615N and 1520518N. Support was also provided by the Simons Foundation for Autism Research Initiative (\#573206). KM was supported by the National Science Foundation Graduate Research Fellowship Program 
bioRxiv preprint doi: https://doi.org/10.1101/2022.02.15.480098; this version posted February 15, 2022. The copyright holder for this preprint (which was not certified by peer review) is the author/funder, who has granted bioRxiv a license to display the preprint in perpetuity. It is made available under aCC-BY 4.0 International license.

bioR $x^{i v}$ Preprint
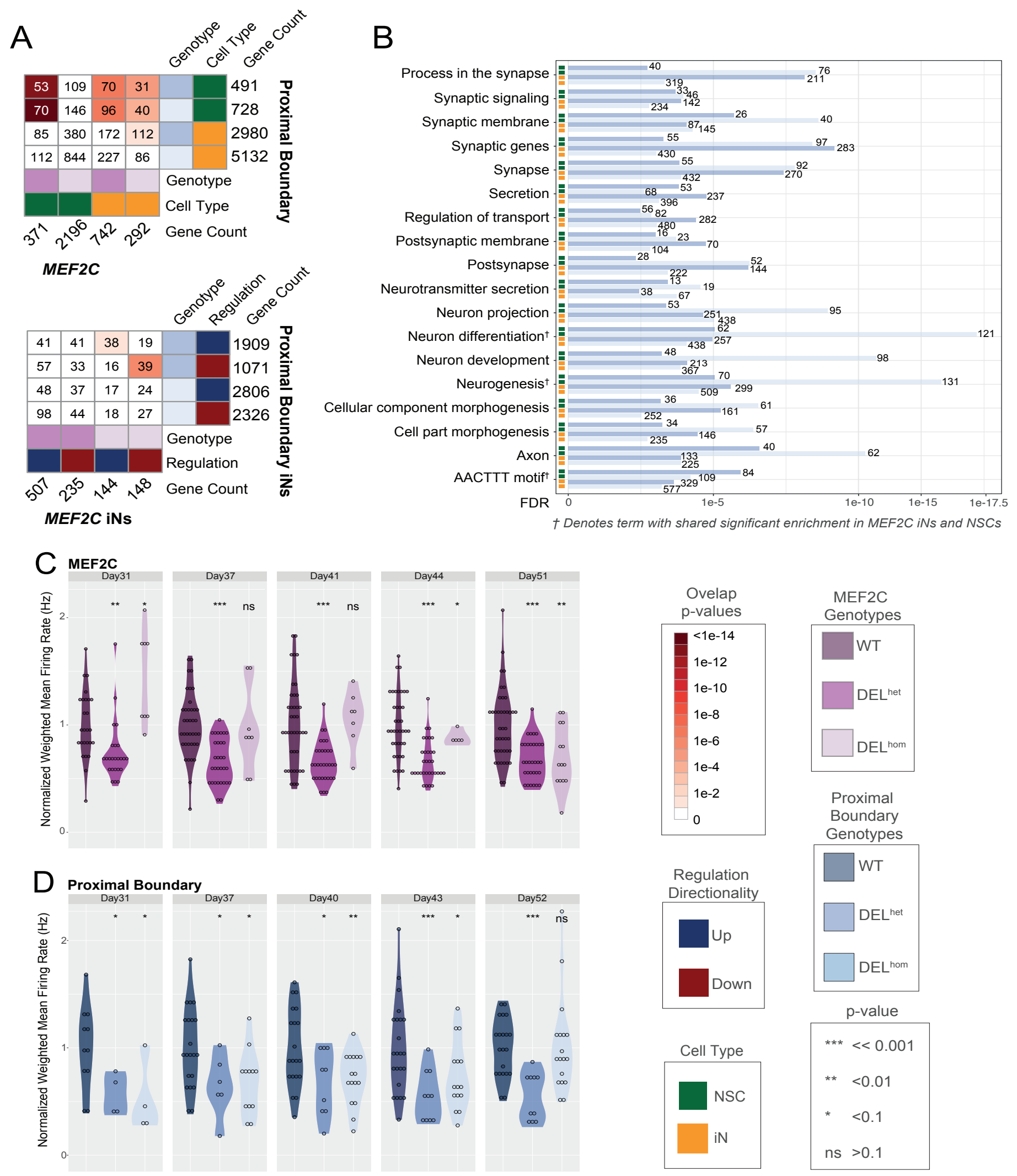

\section{Figure 6 | Proximal Boundary disruption yields disruption of genes involved in neuron differentiation and alters synaptic activity}

A. DEG comparison between Proximal Boundary and MEF2C direct disruption lines (top: both NSCs and iNs, bottom: iNs only). B. Functional enrichment of Proximal Boundary DEGs. Terms shared in MEF2C iNs and NSCs in both genotypes are also noted. Normalized weighted mean firing rate over time as measured by MEA shown for MEF2C iNs (C) and Proximal Boundary iNs (D), respectively. 
bioRxiv preprint doi: https://doi.org/10.1101/2022.02.15.480098; this version posted February $15,2022$. The copyright holder for this preprint (which was not certified by peer review) is the author/funder, who has granted bioRxiv a license to display the preprint in perpetuity. It is made available under aCC-BY 4.0 International license.

bioR ${ }^{\text {iv }}$ Preprint

(NSF GRFP) doctoral fellowship. RY was supported by the Mass General Hospital Fund for Medical Discovery. ED and SV were respectively supported by a doctoral and postdoctoral fellowship of the Research Foundation Flanders (FWO). PB was supported by 1K08NS117891 and T32GM007748. MMO was supported by the Autism Speaks Postdoctoral Fellowship.

\section{AUTHOR CONTRIBUTIONS}

Study design: KM, RY, ED, BM, JFG, SV, MT; Experiments: KM, $\mathrm{ED}, \mathrm{PMB}, \mathrm{MMO}, \mathrm{RB}, \mathrm{BC}, \mathrm{KK}, \mathrm{NB}, \mathrm{DT}, \mathrm{MS}, \mathrm{ML}$. Data analysis: $\mathrm{KM}, \mathrm{RY}, \mathrm{ED}, \mathrm{SE}, \mathrm{DG}, \mathrm{CL}$; Data interpretation: KM, RY, SE, ED, MT; Manuscript writing: KM, RY, SE, ED, JFG, SV, MT. All authors have reviewed and accepted final version of the manuscript.

\section{COMPETING INTERESTS}

M.E.T. receives research funding and/or reagents from Levo Therapeutics, Microsoft Inc, and Illumina Inc. KM initiated employment with Tornado Bio during the writing of this manuscript and is at present an employee of the company. All other authors declare no competing interests. All other authors declare no competing interests.

\section{REFERENCES}

1. Zweier, M. et al. Mutations in MEF2C from the $5 q 14.3 q 15$ microdeletion syndrome region are a frequent cause of severe mental retardation and diminish MECP2 and CDKL5 expression. Human Mutation vol. 31 722-733 (2010).

2. Cardoso, C. et al. Periventricular heterotopia, mental retardation, and epilepsy associated with $5 q 14.3-q 15$ deletion. Neurology 72, 784-792 (2009).

3 . Engels, H. et al. A novel microdeletion syndrome involving 5q14.3-q15: clinical and molecular cytogenetic characterization of three patients. Eur. J. Hum. Genet. 17, 1592-1599 (2009).

4. Novara, F. et al. Refining the phenotype associated withMEF2Chaploinsufficiency. Clinical Genetics vol. 78 471-477 (2010).

5. Novara, F. et al. MEF2C deletions and mutations versus duplications: a clinical comparison. Eur. J. Med. Genet. 56, 260 265 (2013).

6. Nowakowska, B. A. et al. Severe mental retardation, seizures, and hypotonia due to deletions of MEF2C. Am. J. Med. Genet. B Neuropsychiatr. Genet. 153B, 1042-1051 (2010).

7. Le Meur, N., Holder-Espinasse, M. \& Jaillard, S. MEF2C haploinsufficiency caused by either microdeletion of the $5 q 14.3$ region or mutation is responsible for severe mental retardation with stereotypic movements .... Journal of medical (2010).

8. Carr, C. W. et al. $5 q 14.3$ neurocutaneous syndrome: a novel continguous gene syndrome caused by simultaneous deletion of RASA1 and MEF2C. Am. J. Med. Genet. A 155A, 1640-1645 (2011).

9. Tonk, V., Kyhm, J. H., Gibson, C. E. \& Wilson, G. N. Interstitial deletion $5 q 14.3 q 21.3$ with MEF2C haploinsufficiency and mild phenotype: When more is less. American Journal of Medical Genetics Part A vol. 155 1437-1441 (2011).

10. Bienvenu, T., Diebold, B., Chelly, J. \& Isidor, B. Refining the phenotype associated with MEF2C point mutations. Neurogenetics 14, 71-75 (2013).

11. Wang, J. et al. Novel MEF2C point mutations in Chinese patients with Rett (-like) syndrome or non-syndromic intellectual disability: insights into genotype-phenotype correlation. BMC Medical Genetics vol. 19 (2018).

12. Cesaretti, C. et al. Prenatal detection of $5 \mathrm{q} 14.3$ duplication includingMEF2Cand brain phenotype. American Journal of Medical Genetics Part A vol. 170 1352-1357 (2016).
13. Lin, Q., Schwarz, J., Bucana, C. \& Olson, E. N. Control of mouse cardiac morphogenesis and myogenesis by transcription factor MEF2C. Science 276, 1404-1407 (1997).

14. Flavell, S. W. Activity-Dependent Regulation of MEF2 Transcription Factors Suppresses Excitatory Synapse Number. Science vol. 311 1008-1012 (2006).

15. Li, Z. et al. Myocyte Enhancer Factor $2 \mathrm{C}$ as a Neurogenic and Antiapoptotic Transcription Factor in Murine Embryonic Stem Cells. Journal of Neuroscience vol. 28 6557-6568 (2008).

16. Barbosa, A. C. et al. MEF2C, a transcription factor that facilitates learning and memory by negative regulation of synapse numbers and function. Proc. Natl. Acad. Sci. U. S. A. 105, 93919396 (2008).

17. Harrington, A. J. et al. MEF2C Hypofunction in Neuronal and Neuroimmune Populations Produces MEF2C Haploinsufficiency Syndrome-like Behaviors in Mice. Biological Psychiatry vol. 88 488-499 (2020).

18. Neale, B. M. et al. Patterns and rates of exonic de novo mutations in autism spectrum disorders. Nature 485, 242-245 (2012).

19. Hamdan, F. F. et al. High Rate of Recurrent De Novo Mutations in Developmental and Epileptic Encephalopathies. Am. J. Hum. Genet. 101, 664-685 (2017).

20. Coe, B. P. et al. Refining analyses of copy number variation identifies specific genes associated with developmental delay. Nat. Genet. 46, 1063-1071 (2014).

21. Deciphering Developmental Disorders Study. Largescale discovery of novel genetic causes of developmental disorders. Nature 519, 223-228 (2015).

22. Jack M. Fu, F. Kyle Satterstrom, Minshi Peng, Harrison Brand, Ryan L. Collins, Shan Dong, Lambertus Klei, Christine R. Stevens, Caroline Cusick, Mehrtash Babadi, Eric Banks, Brett Collins, Sheila Dodge, Stacey B. Gabriel, Laura Gauthier, Samuel K. Lee, Lindsay Liang, Alicia Ljungdahl, Behrang Mahjani, Laura Sloofman, Andrey Smirnov, Mafalda Barbosa, View ORCID ProfileAlfredo Brusco, Brian H. Y. Chung, Michael L. Cuccaro, Enrico Domenici, Giovanni Battista Ferrero, Jay J. Gargus, Gail E. Herman, Irva Hertz-Picciotto, Patricia Maciel, Dara S. Manoach, Maria Rita Passos-Bueno, Antonio M. Persico, Alessandra Renieri, Flora Tassone, Elisabetta Trabetti, Gabriele Campos, Marcus C. Y. Chan, Chiara Fallerini, Elisa Giorgio, Ana Cristina Girard, Emily Hansen-Kiss, So Lun Lee, Carla Lintas, Yunin Ludena, Rachel Nguyen, Lisa Pavinato, Margaret Pericak-Vance, Isaac Pessah, Evelise Riberi, Rebecca Schmidt, Moyra Smith, Claudia I. C. Souza, Slavica Trajkova, Jaqueline Y. T. Wang, Mullin H. C. Yu, The Autism Sequencing Consoritum (ASC), Broad Institute Center for Common Disease Genomics (BroadCCDG), iPSYCH-BROAD Consortium, David J. Cutler, Silvia De Rubeis, Joseph D. Buxbaum, Mark J. Daly, Bernie Devlin, Kathryn Roeder, Stephan J. Sanders, Michael E. Talkowski. Rare coding variation illuminates the allelic architecture, risk genes, cellular expression patterns, and phenotypic context of autism. MedRxiv. 23. Werling, D. M. et al. An analytical framework for wholegenome sequence association studies and its implications for autism spectrum disorder. Nat. Genet. 50, 727-736 (2018).

24. An, J.-Y. et al. Genome-wide de novo risk score implicates promoter variation in autism spectrum disorder. Science 362, (2018).

25. Aneichyk, T. et al. Dissecting the Causal Mechanism of X-Linked Dystonia-Parkinsonism by Integrating Genome and Transcriptome Assembly. Cell 172, 897-909.e21 (2018).

26. Padhi, E. M. et al. Coding and noncoding variants in EBF3 are involved in HADDS and simplex autism. Hum. Genomics 15, 44 (2021). 
bioRxiv preprint doi: https://doi.org/10.1101/2022.02.15.480098; this version posted February $15,2022$. The copyright holder for this preprint (which was not certified by peer review) is the author/funder, who has granted bioRxiv a license to display the preprint in perpetuity. It is made available under aCC-BY 4.0 International license.

bioR ${ }^{\text {iv }}$ Preprint

27. Oz-Levi, D. et al. Noncoding deletions reveal a gene that is critical for intestinal function. Nature $571,107-111$ (2019).

28. Lieberman-Aiden, E. et al. Comprehensive mapping of long-range interactions reveals folding principles of the human genome. Science 326, 289-293 (2009).

29. Schwarzer, W. et al. Two independent modes of chromatin organization revealed by cohesin removal. Nature 551, 51-56 (2017).

30. Ruiz-Velasco, M. et al. CTCF-Mediated Chromatin Loops between Promoter and Gene Body Regulate Alternative Splicing across Individuals. Cell Syst 5, 628-637.e6 (2017).

31. Rao, S. S. P. et al. A 3D map of the human genome at kilobase resolution reveals principles of chromatin looping. Cell 159, 1665-1680 (2014).

32. Rao, S. S. P. et al. Cohesin Loss Eliminates All Loop Domains. Cell 171, 305-320.e24 (2017).

33. Franke, M. et al. Formation of new chromatin domains determines pathogenicity of genomic duplications. Nature 538, 265-269 (2016).

34. Lupiáñez, D. G. et al. Disruptions of topological chromatin domains cause pathogenic rewiring of geneenhancer interactions. Cell 161, 1012-1025 (2015).

35. Laugsch, M. et al. Modeling the Pathological LongRange Regulatory Effects of Human Structural Variation with Patient-Specific hiPSCs. Cell Stem Cell 24, 736-752.e12 (2019).

36. Ghavi-Helm, Y. et al. Highly rearranged chromosomes reveal uncoupling between genome topology and gene expression. Nat. Genet. 51, 1272-1282 (2019).

37. Despang, A., Schöpflin, R., Franke, M., Ali, S. \& Jerković, I. Functional dissection of the Sox9-Kcnj2 locus identifies nonessential and instructive roles of TAD architecture. Nature (2019).

38. Redin, C. et al. The genomic landscape of balanced cytogenetic abnormalities associated with human congenital anomalies. Nat. Genet. 49, 36-45 (2017).

39. Firth, H. V. richards SM, Bevan aP, clayton S, corpas $\mathrm{M}$, rajan $\mathrm{D}$, Van Vooren $\mathrm{S}$, Moreau $\mathrm{Y}$, Pettett $\mathrm{rM}$, carter $\mathrm{nP}$. Decipher: database of chromosomal imbalance and phenotype in humans using ensembl resources. Am. J. Hum. Genet. 84, 524-533 (2009).

40. Harrington, A. J. et al. MEF2C regulates cortical inhibitory and excitatory synapses and behaviors relevant to neurodevelopmental disorders. eLife vol. 5 (2016).

41. Parikshak, N. N. et al. Integrative functional genomic analyses implicate specific molecular pathways and circuits in autism. Cell 155, 1008-1021 (2013).

42. Li, M. et al. Integrative functional genomic analysis of human brain development and neuropsychiatric risks. Science 362, (2018).

43. Byrne, K. et al. A gene network switch enhances the oxidative capacity of ovine skeletal muscle during late fetal development. BMC Genomics 11, 378 (2010).

44. Bartlett, T. E., Müller, S. \& Diaz, A. Single-cell Coexpression Subnetwork Analysis. Sci. Rep. 7, 15066 (2017).

45. Gómez-Ferrería, M. A. \& Rey-Campos, J. Functional domains of FOXJ2. J. Mol. Biol. 329, 631-644 (2003).

46. Subramanian, A. et al. Gene set enrichment analysis: a knowledge-based approach for interpreting genome-wide expression profiles. Proc. Natl. Acad. Sci. U. S. A. 102, 1554515550 (2005).

47. Liberzon, A. et al. Molecular signatures database
(MSigDB) 3.0. Bioinformatics 27, 1739-1740 (2011).

48. ENCODE Project Consortium. An integrated encyclopedia of DNA elements in the human genome. Nature 489, 57-74 (2012).

49. Karczewski, K. J. et al. The mutational constraint spectrum quantified from variation in 141,456 humans. Nature 581, 434-443 (2020).

50. Abad, $M$. et al. Notch Inhibition Enhances Cardiac Reprogramming by Increasing MEF2C Transcriptional Activity. Stem Cell Reports 8, 548-560 (2017).

51. Vedantham, V., Evangelista, M., Huang, Y. \& Srivastava, D. Spatiotemporal regulation of an Hcn4 enhancer defines a role for Mef2c and HDACs in cardiac electrical patterning. Dev. Biol. 373, 149-162 (2013).

52. Materna, S. C., Sinha, T., Barnes, R. M., Lammerts van Bueren, K. \& Black, B. L. Cardiovascular development and survival require Mef2c function in the myocardial but not the endothelial lineage. Dev. Biol. 445, 170-177 (2019).

53. Mayers, C. M. et al. The Rho guanine nucleotide exchange factor AKAP13 (BRX) is essential for cardiac development in mice. J. Biol. Chem. 285, 12344-12354 (2010).

54. Wang, W. et al. MEF2C protects bone marrow B-lymphoid progenitors during stress haematopoiesis. Nat. Commun. 7, 12376 (2016).

55. Wang, W., Montel-Hagen, A., Sasidharan, R. \& Mikkola, H. K. A. Mef2C maintains $B$ cell homeostasis through the regulation of DNA repair machinery. Blood 120, 278-278 (2012).

56. Franceschini, A. \& Others. STRINGdb Package Vignette. Nucleic Acids Res. (2013).

57. Szklarczyk, D. et al. The STRING database in 2017: qualitycontrolled protein-protein association networks, made broadly accessible. Nucleic Acids Res. 45, D362-D368 (2016).

58. Cline, M. S. et al. Integration of biological networks and gene expression data using Cytoscape. Nat. Protoc. 2, 2366-2382 (2007).

59. Saito, R. et al. A travel guide to Cytoscape plugins. Nat. Methods 9, 1069-1076 (2012).

60. Shannon, P. et al. Cytoscape: a software environment for integrated models of biomolecular interaction networks. Genome Res. 13, 2498-2504 (2003).

61. Lemke, J. R. et al. Mutations in GRIN2A cause idiopathic focal epilepsy with rolandic spikes. Nat. Genet. 45, 1067-1072 (2013).

62. Shi, X. et al. Clinical spectrum of SCN2A mutations. Brain Dev. 34, 541-545 (2012).

63. Salpietro, V. et al. AMPA receptor GluA2 subunit defects are a cause of neurodevelopmental disorders. Nat. Commun. 10, 3094 (2019).

64. Floris, C. et al. Two patients with balanced translocations and autistic disorder: CSMD3 as a candidate gene for autism found in their common 8q23 breakpoint area. Eur. J. Hum. Genet. 16, 696-704 (2008).

65. Saitsu, $H$. et al. De novo $5 \mathrm{q} 14.3$ translocation $121.5-\mathrm{kb}$ upstream of MEF2C in a patient with severe intellectual disability and early-onset epileptic encephalopathy. Am. J. Med. Genet. A 155A, 2879-2884 (2011).

66. Shimojima, K. et al. De novo microdeletion of $5 q 14.3$ excluding MEF2C in a patient with infantile spasms, microcephaly, and agenesis of the corpus callosum. Am. J. Med. Genet. A 158A, 2272-2276 (2012). 67. Yauy, K. et al. Disruption of chromatin organisation causes MEF2C gene overexpression in intellectual disability: a case report. BMC Medical Genomics vol. 12 (2019).

68. Schluth-Bolard, C., Diguet, F. \& Chatron, N. Whole genome paired-end sequencing elucidates functional and phenotypic consequences of balanced chromosomal rearrangement in patients with developmental .... Journal of medical (2019). 
69. Vergult, S. et al. Mate pair sequencing for the detection of chromosomal aberrations in patients with intellectual disability and congenital malformations. Eur. J. Hum. Genet. 22, 652-659 (2014).

70. Dixon, J. R. et al. Topological domains in mammalian genomes identified by analysis of chromatin interactions. Nature 485, 376-380 (2012).

71. D'haene, E. et al. A neuronal enhancer network upstream of MEF2C is compromised in patients with Rett like characteristics. Hum. Mol. Genet. 29, 879-880 (2020).

72. Porter, B. \& Paciorkowski, A. MEF2C haploinsufficiency syndrome: A severe neurodevelopmental disorder with variable epilepsy. J. Pediatr. Epilepsy 01, 171-178 (2015).

73. Paciorkowski, A. R. et al. MEF2C Haploinsufficiency features consistent hyperkinesis, variable epilepsy, and has a role in dorsal and ventral neuronal developmental pathways. Neurogenetics 14, 99-111 (2013).

74. Borlot, F., Whitney, R., Cohn, R. D. \& Weiss, S. K. MEF2C-related epilepsy: Delineating the phenotypic spectrum from a novel mutation and literature review. Seizure 67, 86-90 (2019).

75. Rocha, H., Sampaio, M., Rocha, R., Fernandes, S. \& Leão, M. MEF2C haploinsufficiency syndrome: Report of a new MEF2C mutation and review. Eur. J. Med. Genet. 59, 478-482 (2016).

76. Williamson, I. et al. Developmentally regulated Shh expression is robust to TAD perturbations. Development 146, (2019).

77. Reilly, S. K. et al. Direct characterization of cisregulatory elements and functional dissection of complex genetic associations using HCR-FlowFISH. Nat. Genet. 53, 1166-1176 (2021).

78. Fudenberg, G., Kelley, D. R. \& Pollard, K. S. Predicting 3D genome folding from DNA sequence with Akita. Nat. Methods 17, 1111-1117 (2020).

79. Huynh, L. \& Hormozdiari, F. TAD fusion score: discovery and ranking the contribution of deletions to genome structure. Genome Biol. 20, 60 (2019).

80. Nieboer, M. M. \& de Ridder, J. svMIL: predicting the pathogenic effect of TAD boundary-disrupting somatic structural variants through multiple instance learning. Bioinformatics 36 , i692-i699 (2020). 